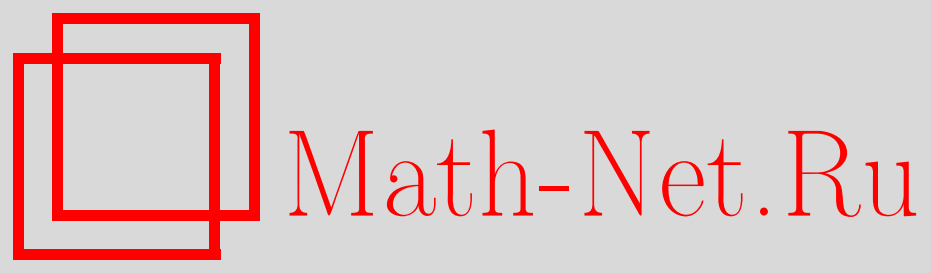

В. П. Елизаров, О классах разрешимых колец, Матем. вопр. криптогр., 2010, том 1, выпуск 3, 19-26

DOI: https://doi.org/10.4213/mvk13

Использование Общероссийского математического портала Math-Net.Ru подразумевает, что вы прочитали и согласны с пользовательским соглашением http://www.mathnet.ru/rus/agreement

Параметры загрузки:

IP : 52.23 .180 .231

26 апреля 2023 г., 18:04:36 
УДК 512.552

\title{
О классах разрешимых колец
}

\author{
Елизаров В. П.
}

ООО «Центр сертификационных исследований», Москва

Получено 22.IV.2010

Описан класс всех колец для систем левых линейных уравнений, над которыми необходимое условие разрешимости - согласованность системы относительно правых идеалов кольца — является достаточным.

Ключевые слова: системы линейных уравнений над кольцами, частные идеалов кольца

\section{On classes of solvable rings}

\section{V.P. Elizarov}

LLC "Certification Research Center", Moscow

\begin{abstract}
We describe the class of all rings such that a system of left linear systems of equations over the ring is solvable iff this system is compatible with respect to the right group ideals.
\end{abstract}

Key words: systems of linear equation over rings, quotients of ideals of a ring

Citation: Mathematical Aspects of Cryptography, 2010, vol. 1, no. 3, pp. 19-26 (Russian). 
Елизаров В. П.

\section{1. Введение}

Термин «кольцо» означает далее ассоциативное кольцо с единицей, а термин «модуль» - унитарный модуль над кольцом. Запись $M_{R}$ или ${ }_{R} M$ показывает, что $M$ - правый или соответственно левый модуль над кольцом $R$. Через $S_{m, n}$ обозначим совокупность всех $m \times n$-матриц с элементами из множества $S$ и в частных случаях полагаем

$$
S_{1, n}=S^{n}, S_{m, 1}=S^{(m)} \text {. }
$$

Подмодуль модуля $M$, порожденный подмножеством $K$, будем обозначать через $\langle K\rangle$.

Если $J$ - правый идеал кольца $R$, то будем писать $J \triangleleft_{r} R$. Запись $T<M$ означает, что $T-$ подмодуль модуля $M$.

Рассматриваются системы линейных уравнений над кольцом $R$ вида

$$
A X^{\downarrow}=B^{\downarrow},
$$

где $A \in R_{m, n}$ и $B^{\downarrow} \in R^{(m)}$. Система уравнений (1) называется разрешимой, если она имеет хотя бы одно решение в $R^{(n)}$.

Имеются различные условия, необходимые для разрешимости системы уравнений (1) над произвольным кольцом $R$. Одно из них - согласованность системы (1) относительно правых идеалов кольца $R$ ( ri-согласованность): для любых $J \triangleleft_{r} R$ и $\vec{C} \in R^{m}$ справедлива импликация

$$
\left(\vec{C} A \in J^{n}\right) \Rightarrow\left(\vec{C} B^{\downarrow} \in J\right) .
$$

Всякая $r i$-согласованная система (1) согласована относительно линейных соотношений ( $l c$-согласована): для любого $\vec{C} \in R^{m}$ справедлива импликация

$$
(\vec{C} A=\overrightarrow{0}) \Rightarrow\left(\vec{C} B^{\downarrow}=0\right) .
$$

Однако в случае произвольного кольца не всякая $l c$-согласованная и даже не всякая $r i$-согласованная система уравнений над ним разрешима (см. [4]).

Кольцо $R$ называется $r i$-разрешимым ( $l c$-разрешимым), если всякая $r i$-согласованная ( $l c$-согласованная) система уравнений вида (1) над $R$ разрешима. Через $S O L(l c)$ и $S O L(r i)$ обозначим классы всех $l c$-разрешимых и всех $r i$-разрешимых колец соответственно. В [6] была поставлена задача описания этих классов.

В [7] дано описание класса $S O L(l c)$ как класса локально замкнутых справа колец и указаны некоторые его подклассы. Относительно класса 
$S O L(r i)$ известно следующее: кольцо $\mathbf{Z}$ целых чисел $r i$-разрешимое [11, $\S 106]$; всякое кольцо, любая матрица над которым эквивалентна линейно ступенчатой (в частности - диагональной) матрице, ri-разрешимое [4].

В настоящей работе дано описание класса $S O L(r i)$. В п. 2 излагается вспомогательный материал: вводятся понятия частных для правых идеалов кольца $R$ по подмножествам из $R^{(n)}$ и $R^{n}, n \in \mathbf{N}$, и рассматриваются свойства частных. В п. 3 в терминах свойств частных для правых идеалов кольца $R$ по конечно порожденным подмодулям из $R_{R}^{(n)}$ дается критерий принадлежности $R$ классу $S O L(r i)$. В п. 4 указаны некоторые подклассы класса $\operatorname{SOL}(r i)$.

\section{2. Частные правых идеалов кольца}

Пусть $R$ - кольцо, $J \triangleleft_{r} R, n \in \mathbf{N}, K \subset R^{(n)}$ и $L \subset R^{n}-$ непустые подмножества.

Определение 1. Левым частным $J$ по $K$ в $R^{n}$ и правым частным $J$ по $L$ в $R^{(n)}$ называются соответственно множества

$$
[J: K]=\left\{\vec{U} \in R^{n} \mid \vec{U} K \subset J\right\}
$$

и

$$
[L: J]=\left\{V^{\downarrow} \in R^{(n)} \mid L V^{\downarrow} \subset J\right\} .
$$

Ясно, что $[J: K]$ - подгруппа группы $\left(R^{n},+\right)$, а $[L: J]<R_{R}^{(n)}$. Понятия левого и правого частных для $J \triangleleft_{r} R$ в $R^{n}$ и $R^{(n)}$ соответственно обобщают, с одной стороны, при $n=1$ понятия частных $J$ по подмножествам из $R$ (см., например [2, § 95]) и, с другой стороны, при $J=\{0\}-$ понятия левого аннулятора $K$ в $R^{n}$ и правого аннулятора $L$ в $R^{(n)}$ (см., например, [9, гл. 3]).

Приведем некоторые свойства частных правых идеалов кольца.

Предложение 1. Пусть $R-$ кольцо, $J \triangleleft_{r} R, n \in N, K$ и $M-$ непустые подмножества из $R^{n}$. Справедливы соотношения:

a) $(K \subset M) \Rightarrow([J: K] \supset[J: M])$;

б) $[J: K]=[J:\langle K\rangle]$;

в) $[[J: K]: J] \supset\langle K\rangle$.

Доказательство предложения 1 стандартно. 
Предложение 2. Пусть $R-$ кольйо, $J \triangleleft_{r} R, n \in N u S_{i}<R_{R}^{(n)}, i \in\{1,2, \ldots, t\}$. Справедливо равенство

$$
\left[J: \sum_{i=1}^{t} S_{i}\right]=\bigcap_{i=1}^{t}\left[J: S_{i}\right]
$$

Доказательство. Так как

$$
S_{j} \subset \sum_{i=1}^{t} S_{i}
$$

при $j \in\{1,2, \ldots, t\}$, то согласно предложению 1 a) получаем

$$
\left[J: S_{j}\right] \supset\left[J: \sum_{i=1}^{t} S_{i}\right]
$$

при $j \in\{1,2, \ldots, t\}$. Значит, справедливо включение

$$
\bigcap_{j=1}^{t}\left[J: S_{j}\right] \supset\left[J: \sum_{i=1}^{t} S_{i}\right] .
$$

Поскольку обратное включение очевидно, то равенство (2) доказано.

Определение 2. Кольцо $R$ назовем (локально) замкнутым относительно частных правых идеалов ((локально) ЧПИ-замкнутым), если для любых $J \triangleleft_{r} R, t \in \mathbf{N}$ и (конечно порожденного) $K<R_{R}^{(t)}$ выполняется равенство

$$
[[J: K]: J]=K \text {. }
$$

Ввиду предложения 1в) определение 2 корректно. Поля дают простейшие примеры ЧПИ-замкнутых колец.

Определение 3. Кольцо $R$ назовем (локально) упорядоченным относительно частных правых идеалов ((локально) ЧПИ-упорядоченным), если для любых $t \in \mathbf{N}$ и (конечно порожденных) $K, M<R_{R}^{(t)}$ справедливо соотношение

$$
(K \subset M) \Leftrightarrow\left(\forall J \triangleleft_{r} R:[J: K] \supset[J: M]\right) .
$$

Предложение 3. Всякое (локально) ЧПИ-замкнутое кольцо $R$ является (локально) ЧПИ-упорядоченныл.

Доказательство. Импликация " $\Rightarrow "$ в (3) справедлива ввиду предложения 1а). Покажем справедливость обратной импликации.

Пусть при любом $J \triangleleft_{r} R$ для (конечно порожденных) $K, M<R_{R}^{(t)}$ имеет место включение

$$
[J: K] \supset[J: M]
$$


Тогда по правому аналогу предложения 1а) при любом $J \triangleleft_{r} R$ справедливо включение

$$
[[J: K]: J] \subset[[J: M]: J] .
$$

Так как $R-$ (локально) ЧПИ-замкнутое кольцо, то из включений (4) следует $K \subset M$. Значит, $R-$ (локально) ЧПИ-упорядоченное кольцо.

\section{3. Описание класса $\operatorname{SOL}(r i)$}

Опишем класс SOL(ri) всех ri-разрешимых колец, т. е. колец, любая $r i$-согласованная система уравнений вида (1) над которыми разрешима. Условие $r i$-согласованности системы (1) можно сформулировать в терминах левых частных правых идеалов кольца $R$.

Предложение 4. Система уравнений (1), где

$$
A=\left(A_{1}^{\downarrow}, \ldots, A_{n}^{\downarrow}\right) \in R_{m, n}
$$

$r i$-согласована тогда и только тогда, когда при любом $J \triangleleft_{r} R$ справедливо включение

$$
\left[J:\left\langle A_{1}^{\downarrow}, \ldots, A_{n}^{\downarrow}\right\rangle\right] \subset\left[J:\left\langle B^{\downarrow}\right\rangle\right] .
$$

Доказательство. Справедливость при любых $J \triangleleft_{r} R$ и $\vec{C} \in R^{m}$ импликации

$$
\left(\vec{C} A \in J^{n}\right) \Rightarrow\left(\vec{C} B^{\downarrow} \in J\right)
$$

равносильна справедливости импликации

$$
\left(\vec{C} \in\left[J: A_{k}^{\downarrow}\right], k \in\{1,2, \ldots, n\}\right) \Rightarrow\left(\vec{C} \in\left[J: B^{\downarrow}\right]\right)
$$

или, согласно предложению 1б), импликации

$$
\left(\vec{C} \in\left[J:\left\langle A_{k}^{\downarrow}\right\rangle\right], k \in\{1,2, \ldots, n\}\right) \Rightarrow\left(\vec{C} \in\left[J:\left\langle B^{\downarrow}\right\rangle\right]\right) .
$$

Таким образом, критерием ri-согласованности системы уравнений (1) является справедливость при любом $J \triangleleft_{r} R$ включения

$$
\bigcap_{k=1}^{n}\left[J:\left\langle A_{k}^{\downarrow}\right\rangle\right] \subset\left[J:\left\langle B^{\downarrow}\right\rangle\right]
$$


которое ввиду предложения 2 можно записать в виде

$$
\left[J: \sum_{k=1}^{n}\left\langle A_{k}^{\downarrow}\right\rangle\right] \subset\left[J:\left\langle B^{\downarrow}\right\rangle\right],
$$

то есть в виде (5).

Теорема 5. Кольцьо $R$ является ri-разрешимым тогда и только тогда, когда оно локально ЧПИ-упорядоченное.

Доказательство. Пусть $R$ - ri-разрешимое кольцо, $t \in \mathbf{N}, \quad K=$ $=\left\langle K_{1}^{\downarrow}, \ldots, K_{u}^{\downarrow}\right\rangle<R_{R}^{(t)}, M=\left\langle M_{1}^{\downarrow}, \ldots, M_{s}^{\downarrow}\right\rangle<R_{R}^{(t)}$ и для любого $J \triangleleft_{r} R$ имеет место включение

$$
[J: K] \supset[J: M]
$$

Учитывая предложение 2, запишем соотношения

$$
\forall J \triangleleft_{r} R\left(\bigcap_{i=1}^{u}\left[J:\left\langle K_{i}^{\downarrow}\right\rangle\right] \supset[J: M]\right) .
$$

Таким образом, при $i \in\{1,2, \ldots, u\}$ справедливы соотношения

$$
\forall J \triangleleft_{r} R\left(\left[J:\left\langle K_{i}^{\downarrow}\right\rangle\right] \supset[J: M]\right) .
$$

Обозначим через $\left(M_{1}^{\downarrow}, \ldots, M_{s}^{\downarrow}\right)$ матрицу, составленную из векторов, порождающих модуль $M$, и рассмотрим системы уравнений

$$
\left(M_{1}^{\downarrow}, \ldots, M_{s}^{\downarrow}\right) X^{\downarrow}=K_{i}^{\downarrow}
$$

где $i \in\{1,2, \ldots, u\}$. Согласно предложению 4 соотношения (7) означают, что все системы уравнений (8) ri-согласованы. По условию они разрешимы. Следовательно, $K_{i}^{\downarrow} \in M$ при $i \in\{1,2, \ldots, u\}$ и поэтому $K \subset M$. Значит, $R$ локально ЧПИ-упорядоченное кольцо.

Обратно, пусть $R$ - локально ЧПИ-упорядоченное кольцо и система уравнений (1) ri-согласована. По предложению 4 при любом $J \triangleleft_{r} R$ справедливо включение (5), из которого по определению 3 получаем включение

$$
\left\langle A_{1}^{\downarrow}, \ldots, A_{n}^{\downarrow}\right\rangle \supset\left\langle B^{\downarrow}\right\rangle,
$$

означающее разрешимость системы уравнений (1). Стало быть, $R$ $r i$-разрешимое кольцо. 


\section{4. Некоторые классы $r i$-разрешимых колец}

Укажем некоторые подклассы класса $\mathrm{SOL}(r i)$.

Очевидно, справедливо следующее утверждение.

Предложение 6. Имеет место соотношение

$$
\mathrm{SOL}(l c) \subset \mathrm{SOL}(r i) .
$$

Напомним описание класса $\operatorname{SOL}(l c)$.

Определение 5. Кольцо $R$ называется локально замкнутым справа, если для любого $n \in \mathbf{N}$ и любого конечно порожденного $K<R_{R}^{(n)}$ выполняется равенство

$$
\operatorname{Ann}_{R^{(n)}} \operatorname{Ann}_{R^{n}} K=K,
$$

то есть равенство $[[0: K]: 0]=K$.

Предложение 7. Класс $\mathrm{SOL}(l c)$ - это в точности класс всех локально замкнутых справа колеи [7, теорема 3].

Предложение 8. Класс локально ЧПИ-замкнутых колеи является подклассом класса $\mathrm{SOL}($ ri) .

Доказательство. Согласно предложению 3 локально ЧПИ-замкнутое кольцо $R$ является локально ЧПИ-упорядоченным. По теореме 5 оно $r i$-разрешимое.

Пусть $R$ - кольцо. Матрицы $A, F \in R_{m, n}$ называются эквивалентными, если существуют такие обратимые матрицы $U \in R_{m, m}$ и $V \in R_{n, n}$, что выполняется равенство $F=U A V$ (см. [1, гл. $2, \S 6]$ ).

Определение 6. Кольцо $R$ назовем диагональным, если любая матрица над $R$ эквивалентна некоторой диагональной матрице. Класс всех диагональных колец обозначим через DIAG .

Предложение 9. Имеет место соотночение

$$
\mathrm{DIAG} \subset \mathrm{SOL}(r i) .
$$

Доказательство. Пусть $R \in$ DIAG. По теореме 8 из [4] всякая $r i$-согласованная система уравнений над $R$ вида (1) разрешима. Значит, $R-r i$-разрешимое кольцо.

Диагональным кольцом является, например, всякая область целостности главных правых и главных левых идеалов (см. [10] или [3, гл. 3]). Большое число примеров таких областей приведено в [8]. Ясно, что класс DIAG замкнут относительно прямых сумм и гомоморфных образов. 
Елизаров В. П.

В заключение отметим, что понятия $l c$-согласованности и $r i$-согласованности введены в [5] и для систем линейных уравнений над левыми $R$-модулями. В [7] описан класс $l c$-разрешимых $R$-модулей. Большинство из приведенных выше результатов может быть перенесено на класс $r i$-разрешимых $R$-модулей, но это предмет отдельной работы.

\section{Список литературы}

1. Бурбаки Н. Алгебра: алгебраические структуры, линейная и полилинейная алгебра. - М.: ФИЗМАТГИЗ, 1962.

2. Ван дер Варден Б. Л. Алгебра. - М.: Наука, 1976.

3. Джекобсон Н. Теория колец. - М.: ГИИЛ, 1947.

4. Елизаров В.П. Условия совместности систем линейных уравнений над кольцами. - Фундам. и прикл. матем., 2000, т. 6, № 3, с. 777-788.

5. Елизаров В. П. Системы линейных уравнений над модулями. - Фундам. и прикл. матем., 2002, т. 8, № 4, с. 979-991.

6. Елизаров В. П. Условия, необходимые для разрешимости системы линейных уравнений над кольцом. - Дискретн. матем., 2004, т. 16, в. 2, c. 44-53.

7. Елизаров В. П. Разрешимые и локально замкнутые модули и кольца. Дискрет. матем., 2006, т. 18, в. 1, с. 30-39.

8. Родосский К. А. Алгоритм Евклида. - М.: Наука, 1988.

9. Фейс К. Алгебра: кольца, модули и категории. Т. 1. - М.: Мир, 1977.

10. Asano $K$. Über Verallgemeinerte Abelsche Gruppen mit hypercomplexem Operatorenring und ihre Anwendungen. - Jap. J. Math., 1939, v. 15, N. 4, p. 231-253.

11. Van der Waerden B. L. Moderne Algebra. Teil II. — Berlin: Springer, 1931. 